\title{
Distribution of constant terms of irreducible polynomials in $\mathbb{Z}_{p}[x]$
}

\section{Sarah C. Cobb ${ }^{1}$, Michelle L. Knox ${ }^{2}$, Marcos Lopez ${ }^{3}$, Terry McDonald ${ }^{4}$ and Patrick Mitchell ${ }^{5}$}

\author{
Department of Mathematics, Midwestern State University \\ 3410 Taft Blvd, Wichita Falls, TX 76308 USA \\ e-mails: ${ }^{1}$ sarah.cobb@msutexas . edu, ${ }^{2}$ michelle. knox@msutexas . edu, \\ ${ }^{3}$ marcos.lopez@msutexas.edu, ${ }^{4}$ terry.mcdonald@msutexas.edu, \\ ${ }^{5}$ patrick.mitchell@msutexas.edu
}

Received: 29 May 2019

Accepted: 12 September 2019

\begin{abstract}
We obtain explicit formulas for the number of monic irreducible polynomials with prescribed constant term and degree $q^{k}$ over a finite field. These formulas are derived from work done by Yucas. We show that the number of polynomials of a given constant term depends only on whether the constant term is a residue in the underlying field. We further show that as $k$ becomes large, the proportion of irreducible polynomials having each constant term is asymptotically equal.
\end{abstract}

Keywords: Irreducible polynomials, Finite fields.

2010 Mathematics Subject Classification: 11T06, $12 \mathrm{E} 05$.

\section{Introduction}

The distribution of primes across equivalence classes in modular arithmetic is a well-studied problem in number theory. According to Dirichlet's Theorem, the proportion of primes in each equivalence class for a given modulus is asymptotically equal. When only primes less than some finite bound are considered, however, there are usually more primes of the form $4 n+3$ than of the form $4 n+1$, a phenomenon known as Chebyshev's bias. Rubinstein and Sarnak show in [4] that, assuming the Generalized Riemann Hypothesis, this bias generalizes to other moduli: for a fixed $k$, primes of the form $k n+a$ are more common when $a$ is not a quadratic residue mod $k$ than when it is. 
In this paper, we will show that a related bias holds for monic irreducible polynomials over $\mathbb{Z}_{p}$ whose degree is $q^{k}$ for some odd prime $q$. In this case, the number of monic irreducible polynomials with a given constant term $a$ is related to whether $a$ is a residue in the underlying field. As the degree grows larger, however, the proportion of such polynomials ending in each possible constant term is asymptotically equal.

Throughout this paper, $p$ and $q$ are assumed to be odd primes, $\phi$ denotes the Euler phi function, and $\Phi_{n}$ denotes the $n$th cyclotomic polynomial. Much of the other notation follows Yucas in [5].

Let $N(n, a, p)$ denote the number of monic irreducible polynomials over $\mathbb{Z}_{p}$ of degree $n$ with constant term $(-1)^{n} a$. We limit our discussion to polynomials where the degree is a power of an odd prime. To establish a formula for $N(n, a, p)$, Yucas considers the possible orders of irreducible polynomials. For $n \in \mathbb{N}$, define a set

$$
D_{n}=\left\{r: r \mid p^{n}-1 \text { but } r \nmid p^{m}-1 \text { for } 1 \leq m<n\right\} .
$$

Note that $D_{n}$ is the set of possible orders of polynomials of degree $n$ over $\mathbb{Z}_{p}^{*}$. For any $r \in D_{n}$, we can write $r=d_{r} m_{r}$ where $d_{r}=\operatorname{gcd}\left(r, \frac{p^{n}-1}{p-1}\right)$. When $n$ is a power of a prime, we have the following characterization of $D_{n}$ :

Lemma 1.1. Let $n=q^{k}$ for some $k \in \mathbb{N}$, then

$$
D_{n}=\left\{r: r \mid p^{q^{k}}-1 \text { but } r \nmid p^{q^{k-1}}-1\right\} .
$$

Proof. Note that $\operatorname{gcd}\left(p^{q^{k}}-1, p^{m}-1\right)=p^{\operatorname{gcd}\left(q^{k}, m\right)}-1\left(\right.$ see Lemma 12.6 in [1]). If $\operatorname{gcd}\left(q^{k}, m\right)=1$ and $r \in D_{n}$ with $r \mid p^{m}-1$, then $r \mid p-1$. Otherwise, $r \mid p^{m}-1$ for some divisor $m$ of $q^{k}$, i.e., $r \mid p^{q^{i}}$ for some $0 \leq i<k$. But $p^{q^{i}}-1$ divides $p^{q^{k-1}}-1$ for any $0 \leq i \leq k-1$.

Lemma 1.1 allows us to focus our attention on divisors of $p^{q^{k-1}}-1$ instead of looking for all possible values of $m$ where $r \mid p^{m}-1$. Using this set $D_{n}$ and the order of the element $a \in \mathbb{Z}_{p}^{*}$, Yucas derives the following formula for $N(n, a, p)$ :

Theorem 1.2 ([5, Theorem 3.5]). Suppose $a \in \mathbb{Z}_{p}^{*}$ has order m. Then

$$
N(n, a, p)=\frac{1}{n \phi(m)} \sum_{\substack{r \in D_{n} \\ m_{r}=m}} \phi(r) .
$$

While this gives a method for computing $N(n, a, p)$ in any case, it does not provide a clear way to compare different cases. Our goal is to establish the distribution of constant terms for a fixed $p$ and $q^{k}$ for $k \in \mathbb{N}$. This depends on the distribution of $q$ th powers in $\mathbb{Z}_{p}^{*}$.

Definition 1.3. Let $a \in \mathbb{Z}_{p}^{*}$. If there is some $b \in \mathbb{Z}_{p}^{*}$ such that $b^{q} \equiv a(\bmod p)$, then $a$ is $a$ $q$-residue in $\mathbb{Z}_{p}^{*}$.

As we see in Theorem 1.4, the distribution of $q$-residues in $\mathbb{Z}_{p}^{*}$ depends on whether $q$ divides $p-1$, which allows us to determine the number of $q$-residues in $\mathbb{Z}_{p}^{*}$ in Proposition 1.5. 
Theorem 1.4 ([3, Theorem 2.37]). If $p$ is a prime and $\operatorname{gcd}(a, p)=1$, then the congruence $x^{n} \equiv a(\bmod p)$ has $\operatorname{gcd}(n, p-1)$ solutions or no solution according as $a^{\frac{p-1}{\operatorname{gcd}(n, p-1)}} \equiv 1(\bmod p)$ or not.

Proposition 1.5. If $\operatorname{gcd}(q, p-1)=q$, then there are $\frac{p-1}{q} q$-residues in $\mathbb{Z}_{p}^{*}$. Otherwise, every element of $\mathbb{Z}_{p}^{*}$ is a q-residue.

Proof. Observe that $\operatorname{gcd}(a, p)=1$ for every $a \in \mathbb{Z}_{p}^{*}$. If $\operatorname{gcd}(q, p-1)=q$, then $q \mid p-1$. By Theorem 1.4, for any $a \in \mathbb{Z}_{p}^{*}, x^{q} \equiv a(\bmod p)$ has $\operatorname{gcd}(q, p-1)=q$ solutions or no solutions. Hence $\frac{p-1}{q}$ values of $a$ have a solution to that equation. If $\operatorname{gcd}(q, p-1)=1$, then $a^{\frac{p-1}{1}} \equiv 1(\bmod p)$ because $\mathbb{Z}_{p}^{*}$ has $p-1$ elements. So every $a \in \mathbb{Z}_{p}^{*}$ is a $q$-residue.

In Section 2, we will consider the case where $\operatorname{gcd}(q, p-1)=1$. We will prove that for any $a \in \mathbb{Z}_{p}^{*}$

$$
N\left(q^{k}, a, p\right)=\frac{p^{q^{k}}-p^{q^{k-1}}}{q^{k}(p-1)} .
$$

In the case where $\operatorname{gcd}(q, p-1)=q$, the value of $N\left(q^{k}, a, p\right)$ depends on whether or not $a$ is a $q$-residue in $\mathbb{Z}_{p}^{*}$. We will address this in Sections 3 and 4 . In particular, we will show that

$$
N\left(q^{k}, a, p\right)=\frac{p^{q^{k}}-1}{q^{k}(p-1)}
$$

whenever $a$ is not a $q$-residue in $\mathbb{Z}_{p}^{*}$ and

$$
N\left(q^{k}, a, p\right)=\frac{p^{q^{k}}-q p^{q^{k-1}}+q-1}{q^{k}(p-1)}
$$

whenever $a$ is a $q$-residue in $\mathbb{Z}_{p}^{*}$.

In Yucas's formula, $N\left(q^{k}, a, p\right)$ represents the number of irreducible monic polynomials with a constant term of $(-1)^{q^{k}} a$. In our case, we assume $q$ is an odd prime, hence $N\left(q^{k}, a, p\right)$ is the number of monic irreducible polynomials with a constant term of $-a$. Since $a$ is a $q$-residue if and only if $-a$ is a $q$-residue, $N\left(q^{k}, a, p\right)$ is the number of irreducible monic polynomials with constant term either $a$ or $-a$.

\section{A formula for $N\left(q^{k}, a, p\right)$ when $\operatorname{gcd}(q, p-1)=1$}

Before we can compute $N\left(q^{k}, a, p\right)$ when $\operatorname{gcd}(q, p-1)=1$, we need to present some ancillary results. Recall that $r=d_{r} m_{r}$ where $d_{r}=\operatorname{gcd}\left(r, \frac{p^{n}-1}{p-1}\right)$ and $m_{r}$ is the order of $r$ in $\mathbb{Z}_{p}^{*}$.

Lemma 2.1. Let $r \in D_{n}$. Then $r \mid \frac{p^{n}-1}{p-1}$ if and only if $m_{r}=1$.

Proof. If $r$ divides $\frac{p^{n}-1}{p-1}$, then $d_{r}=r$ implies $m_{r}=1$. Conversely, $m_{r}=1$ implies $r=d_{r}$ and thus $r$ divides $\frac{p^{n}-1}{p-1}$. 
Theorem 2.2. Let $n=q^{k}$ for some $k \in \mathbb{N}$, and let $R_{1}=\left\{r \in D_{n}: m_{r}=1\right\}$. Then

$$
R_{1}=\left\{r \in \mathbb{N}: r \mid \frac{p^{q^{k}}-1}{p-1} \text { and } r \nmid p^{q^{k-1}}-1\right\} \text {. }
$$

Proof. Let $S=\left\{r \in \mathbb{N}: r \mid \frac{p^{q^{k}}-1}{p-1}\right.$ and $\left.r \nmid p^{q^{k-1}}-1\right\}$. Let $r \in R_{1}$, then $m_{r}=1$ implies $r \mid \frac{p^{q^{k}}-1}{p-1}$ by Lemma 2.1. By the definition of $D_{n}, r$ does not divide $p^{m}-1$ for any $1 \leq m<n$ and hence $r \nmid p^{q^{k-1}}-1$. So $r \in S$ and $R_{1} \subseteq S$.

Next suppose $r \in S$. By Lemma 1.1, $r \in D_{n}$, and $m_{r}=1$ by Lemma 2.1. Thus, $S \subseteq R_{1}$.

Corollary 2.2.1. Let $k \in \mathbb{N}, n=q^{k}$, and $\operatorname{gcd}(q, p-1)=1$. For any $r \in D_{n}, d_{r} \in R_{1}$.

Proof. Since $r \in D_{n}$ with order $m_{r}, r \nmid p^{q^{k-1}}-1$, say $t$ is a prime dividing $r$ but not $p^{q^{k-1}}-1$. If $t \mid m_{r}$, then $t \mid p-1$ which means $t \mid p^{q^{k-1}}-1$, a contradiction. So $t \mid d_{r}$, thus $d_{r} \nmid p^{q^{k-1}}-1$. By definition of $d_{r}, d_{r} \mid \frac{p^{q^{k}}-1}{p-1}$, hence $d_{r} \in R_{1}$.

Lemma 2.3. For $i \in \mathbb{N} \operatorname{gcd}\left(\Phi_{q}\left(p^{i}\right), p-1\right) \leq q$.

Proof. Let $s=\operatorname{gcd}\left(\Phi_{q}\left(p^{i}\right), p-1\right)$. Then, we can write $p-1=s t$ for some $t \in \mathbb{N}$. It follows that

$$
\Phi_{q}\left(p^{i}\right)=\Phi_{q}\left((s t+1)^{i}\right)=(s t+1)^{i(q-1)}+(s t+1)^{i(q-2)}+\ldots+(s t+1)^{i}+1 .
$$

Expanding this expression yields $q$ ones, and since $s$ divides the remaining terms on that side of the equation as well as $\Phi_{q}\left(p^{i}\right), s \mid q$.

Lemma 2.4. For $k \in \mathbb{N}$,

$$
\operatorname{gcd}\left(\frac{p^{q^{k}}-1}{p-1}, p^{q^{k-1}}-1\right)=\left\{\begin{array}{cl}
q \cdot \frac{p^{q^{k-1}}-1}{p-1} & \text { if } \operatorname{gcd}(q, p-1)=q \\
\frac{p^{q^{k-1}}-1}{p-1} & \text { if } \operatorname{gcd}(q, p-1)=1
\end{array} .\right.
$$

Proof. Observe that $p^{q^{k}}-1=(p-1) \prod_{i=0}^{k-1} \Phi_{q}\left(p^{q^{i}}\right)$. Hence

$$
\begin{aligned}
\operatorname{gcd}\left(\frac{p^{q^{k}}-1}{p-1}, p^{q^{k-1}}-1\right) & =\operatorname{gcd}\left(\prod_{i=0}^{k-1} \Phi_{q}\left(p^{q^{i}}\right),(p-1) \prod_{i=0}^{k-2} \Phi_{q}\left(p^{q^{i}}\right)\right) \\
& =\left[\prod_{i=0}^{k-2} \Phi_{q}\left(p^{q^{i}}\right)\right] \operatorname{gcd}\left(\Phi_{q}\left(p^{q^{k-1}}\right), p-1\right) \\
& =\left[\frac{p^{q^{k-1}}-1}{p-1}\right] \operatorname{gcd}\left(\Phi_{q}\left(p^{q^{k-1}}\right), p-1\right) .
\end{aligned}
$$

By Lemma 2.3, $\operatorname{gcd}\left(\Phi_{q}\left(p^{q^{k-1}}\right), p-1\right)$ equals 1 or $q$ depending on whether $q$ divides $p-1$.

Corollary 2.4.1. For $k \in \mathbb{N}$, if $\operatorname{gcd}(q, p-1)=1$, then $\operatorname{gcd}\left(\frac{p^{q^{k}}-1}{p-1}, p-1\right)=1$. If $\operatorname{gcd}(q, p-1)=q$, then $q$ is the only prime divisor of $\operatorname{gcd}\left(\frac{p^{q^{k}}-1}{p-1}, p-1\right)$. 
Proof. The results follow from the previous two lemmas and the fact that

$$
p^{q^{k}}-1=(p-1) \prod_{i=0}^{k-1} \Phi_{q}\left(p^{q^{i}}\right) .
$$

Theorem 2.5. Let $k \in \mathbb{N}, \operatorname{gcd}(q, p-1)=1$, and $a \in \mathbb{Z}_{p}^{*}$, then

$$
N\left(q^{k}, a, p\right)=\frac{p^{q^{k}}-q p^{q^{k-1}}}{q^{k}(p-1)} .
$$

Proof. Let $n=q^{k}$ and $a$ have order $m$. By [5, Theorem 3.5], we have

$$
N\left(q^{k}, a, p\right)=\frac{1}{q^{k} \phi(m)} \sum_{\substack{r \in D_{n} \\ m_{r}=m}} \phi(r) .
$$

For any $r \in D_{n}$ with $m_{r}=m$, we can write $r=m_{r} d_{r}$ with $\operatorname{gcd}\left(m_{r}, d_{r}\right)=1$ by Corollary 2.4.1. Thus, we have

$$
N\left(q^{k}, a, p\right)=\frac{1}{q^{k} \phi(m)} \sum_{\substack{r \in D_{n} \\ m_{r}=m}} \phi\left(m_{r}\right) \phi\left(d_{r}\right) .
$$

Recalling that $\sum_{d \mid n} \phi(d)=n$, we use Corollary 2.2.1 and properties of the Euler $\phi$ function to get

$$
N\left(q^{k}, a, p\right)=\frac{1}{q^{k}} \sum_{\substack{d_{r} \mid \frac{p^{n}-1}{p-1} \\ d_{r} \nmid p^{k-1}-1}} \phi\left(d_{r}\right)=\frac{1}{q^{k}}\left[\sum_{d_{r} \mid \frac{p^{n}-1}{p-1}} \phi\left(d_{r}\right)-\sum_{d_{r} \mid \operatorname{gcd}\left(\frac{p^{n}-1}{p-1}, p^{q^{k-1}}-1\right)} \phi\left(d_{r}\right)\right]
$$

From Lemma 2.4 we know

$$
\operatorname{gcd}\left(\frac{p^{q^{k}}-1}{p-1}, p^{q^{k-1}}-1\right)=\frac{p^{q^{k-1}}-1}{p-1}
$$

thus

$$
\begin{aligned}
N\left(q^{k}, a, p\right) & =\frac{1}{q^{k}}\left[\frac{p^{q^{k}}-1}{p-1}-\frac{p^{q^{k-1}}-1}{p-1}\right] \\
& =\frac{p^{q^{k}}-1-\left(p^{q^{k-1}}-1\right)}{q^{k}(p-1)} \\
& =\frac{p^{q^{k}}-q p^{q^{k-1}}}{q^{k}(p-1)} .
\end{aligned}
$$

\section{$3 \quad$ Results when $\operatorname{gcd}(q, p-1)=q$ and $a$ is not a $q$-residue}

When $\operatorname{gcd}(q, p-1)=q, \mathbb{Z}_{p}^{*}$ contains non $q$-residues as well as $q$-residues. The value of $N\left(q^{k}, a, p\right)$ depends on whether or not $a$ is a $q$-residue. In this section, we will prove $N\left(q^{k}, a, p\right)=\frac{p^{q^{k}}-1}{q^{k}(p-1)}$ when $a$ is not a $q$-residue. Theorem 3.1 is important in proving this result, since it classifies the maximum power of $q$ dividing $m_{r}$ when $r$ is not a $q$-residue. 
Theorem 3.1. Let $\mathbb{Z}_{p}^{*}=\langle a\rangle$ and let $p-1=q^{i}$ s for some integer $s$ with $\operatorname{gcd}(q, s)=1$ and some $i \in \mathbb{N}$. Let $b=a^{k}$ for some $k \in \mathbb{Z}$ with the order of b being $m_{b}$. The following are equivalent.

1. $b$ is not a q-residue.

2. $q^{i} \mid m_{b}$

3. $q \nmid \operatorname{gcd}(k, p-1)$.

Proof. First, we will show $(1) \Rightarrow(2)$. Assume $q^{i} \nmid m_{b}$, then $m_{b}=q^{j} t$ for some $0 \leq j<i$ and integer $t$ dividing $s\left(\right.$ since $\left.m_{r} \mid p-1\right)$ with $\operatorname{gcd}(q, t)=1$. Notice

$$
a^{p-1} \equiv 1 \equiv b^{m_{b}} \equiv a^{m_{b} k}(\bmod p)
$$

So, $p-1 \mid m_{b} k$, that is, $\left(q^{i} s\right) \mid\left(q^{j} t k\right)$ where $j<i$, hence $q^{i-j} \mid k$, say $k=q^{i-j} u$ for some integer $u$. It follows that

$$
b=a^{k}=a^{q^{i-j} u}=\left(a^{q^{i-j-1} u}\right)^{q}
$$

is a $q$-residue.

Next, we will prove $(2) \Rightarrow(3)$. Assume $q^{i} \mid m_{b}$, then $m_{b}=q^{i} t$ for some integer $t$ dividing $s$ with $\operatorname{gcd}(q, t)=1$. It follows that

$$
\left|a^{k}\right|=|b|=m_{b}=q^{i} t=\frac{p-1}{\operatorname{gcd}(k, p-1)}=\frac{q^{i} s}{\operatorname{gcd}(k, p-1)}
$$

and thus $q \nmid \operatorname{gcd}(k, p-1)$.

Finally, to show that $(3) \Rightarrow(1)$, assume $b$ is a $q$-residue, say $b=a^{k}=a^{q m}$ for some $m \in \mathbb{Z}$. Then $p-1 \mid(k-q m)$ implies $(p-1) u=k-q m$ for some $u \in \mathbb{Z}$. Note $q^{i} s u=k-q m$ implies $k=q^{i} s u+q m$. Since $p-1$ and $k$ are both divisible by $q$, so is $\operatorname{gcd}(k, p-1)$.

Theorem 3.2. Let $k \in \mathbb{N}, \operatorname{gcd}(q, p-1)=q$, and let $a \in \mathbb{Z}_{p}^{*}$ be a non $q$-residue. Then,

$$
N\left(q^{k}, a, p\right)=\frac{p^{q^{k}}-1}{q^{k}(p-1)} .
$$

Proof. Let $n=q^{k}$ and $r \in D_{n}$. Let $p-1=q^{i} s$ for some integer $s$ with $\operatorname{gcd}(s, q)=1$ and $i \in \mathbb{N}$. Since $a$ is not a $q$-residue, and since $m_{r} \mid p-1$, by Theorem 3.1, $m_{r}=q^{i} v$ for some integer $v$ such that $v \mid s$ and with $\operatorname{gcd}(v, q)=1$. We can also write $\frac{p^{q^{k}}-1}{p-1}=q^{j} t$ for some integer $t$ with $\operatorname{gcd}(q, t)=1$ and $j \in \mathbb{N}$. We claim that $\operatorname{gcd}(v, t)=1$. By Corollary 2.4.1, if $\operatorname{gcd}(p, q-1)=q$, then $q$ is the only prime divisor of

$$
\operatorname{gcd}\left(\frac{p^{q^{k}}-1}{p-1}, p-1\right)=\operatorname{gcd}\left(q^{j} t, q^{i} s\right) .
$$

Since $m_{r}$ divides $p-1$, then $q$ must also be the only prime divisor of $\operatorname{gcd}\left(q^{j} t, q^{i} v\right)$. We note that since $\operatorname{gcd}(v, q)=\operatorname{gcd}(t, q)=1$, and that $q$ must be the only divisor of $\operatorname{gcd}\left(q^{j} t, q^{i} v\right)$, then we must have $\operatorname{gcd}(v, t)=1$. 
We claim that $r=q^{i+j} v u$ for some $u$ that divides $t$. Recall $r=m_{r} d_{r}$ where $d_{r}=\operatorname{gcd}\left(r, \frac{p^{q^{k}}-1}{p-1}\right)$, and we have assumed $m_{r}=q^{i} v$. Since $m_{r}$ has $q^{i}$ as a factor, then $d_{r}$ must have $q^{j}$ as a factor as well. The reasoning for this is if $d_{r}=q^{\ell} u$ with $\operatorname{gcd}(q, u)=1$ and $\ell<j$, then

$$
d_{r}=\operatorname{gcd}\left(r, \frac{p^{q^{k}}-1}{p-1}\right)=\operatorname{gcd}\left(m_{r} d_{r}, q^{j} t\right)=\operatorname{gcd}\left(\left(q^{i} v\right)\left(q^{\ell} u\right), q^{j} t\right)=q^{\ell} u
$$

This implies that $u$ must divide $t$. Observe that $j \geq \ell+1$ and $i+\ell \geq \ell+1$ (because $i \neq 0)$, hence $\operatorname{gcd}\left(\left(q^{i} v\right)\left(q^{\ell} u\right), q^{j} t\right)$ should be divisible by $q^{\ell+1}$, contradicting our assumption that $d_{r}=q^{\ell} u$. Thus, $q^{j} \mid d_{r}$, and we can write $d_{r}=q^{j} u$ for some integer $u$ which divides $t$ and where $\operatorname{gcd}(q, t)=1$. It follows that $r=m_{r} d_{r}=\left(q^{i} v\right)\left(q^{j} u\right)=q^{i+j} v u$ where $u \mid t$. Note that Corollary 2.4.1 implies that $\operatorname{gcd}(s, t)=1$. Thus, $\operatorname{gcd}(u, v)=1$ since $u \mid t$ and $v \mid s$.

Now we can prove the theorem. By [5, Theorem 3.5], we have

$$
N\left(q^{k}, a, p\right)=\frac{1}{q^{k} \phi(m)} \sum_{\substack{r \in D_{n} \\ m_{r}=m}} \phi(r) .
$$

The previous paragraph allows us to write

$$
N\left(q^{k}, a, p\right)=\frac{1}{q^{k} \phi\left(q^{i}\right) \phi(v)} \sum_{\substack{r \in D_{n} \\ u \mid t}} \phi\left(q^{i+j} v u\right) .
$$

We can rewrite the $\phi(r)$ from this expression as $\phi\left(q^{i+i}\right) \phi(v) \phi(u)$ since

$$
\operatorname{gcd}(v, q)=\operatorname{gcd}(u, q)=\operatorname{gcd}(v, u)=\operatorname{gcd}(v, t)=\operatorname{gcd}(q, t)=1 .
$$

Now such an $r$ from $D_{n}$ cannot divide $p^{m}-1$ for any $m<q^{k}$, but Lemma 1.1 implies we need only check for divisors that come from $p^{q^{k-1}}-1$. In this case, the fact that $q^{i+j}$ divides $r$ and

$$
p^{q^{k}}-1=\left(\frac{p^{q^{k}}-1}{p-1}\right)(p-1)=\left(q^{j} t\right)\left(q^{i} s\right)=q^{i+j} s t
$$

prevents $r$ from dividing $p^{q^{\ell}}-1$ when $\ell<k$. Hence we can say

$$
N\left(q^{k}, a, p\right)=\frac{1}{q^{k} \phi\left(q^{i}\right) \phi(v)} \sum_{u \mid t} \phi\left(q^{i+j}\right) \phi(v) \phi(u) .
$$

Using properties of the Euler $\phi$ function, we get

$$
\begin{aligned}
N\left(q^{k}, a, p\right) & =\frac{\phi\left(q^{i+j}\right) \phi(v)}{q^{k} \phi\left(q^{i}\right) \phi(v)} \sum_{u \mid t} \phi(u) \\
& =\frac{q^{i+j}-q^{i+j-1}}{q^{k}\left(q^{i}-q^{i-1}\right)} \sum_{u \mid t} \phi(u) \\
& =\frac{q^{i+j-1}(q-1)}{q^{k} q^{i-1}(q-1)} \sum_{u \mid t} \phi(u) \\
& =\frac{q^{j} t}{q^{k}} \\
& =\frac{p^{q^{k}}-1}{q^{k}(p-1)} .
\end{aligned}
$$




\section{Results when $\operatorname{gcd}(q, p-1)=q$ and $a$ is a $q$-residue}

In Section 3, we were able to directly compute $N\left(p^{k}, a, p\right)$ when $\operatorname{gcd}(q, p-1)=q$ and $a$ is not a $q$-residue. In order to compute $N\left(q^{k}, a, p\right)$ when $\operatorname{gcd}(q, p-1)=q$ and $a$ is a $q$-residue, we will first compute $N\left(q^{k}, 1, p\right)$. We will then prove that $N\left(q^{k}, a, p\right)=N\left(q^{k}, 1, p\right)$ whenever $a$ is a $q$-residue.

Theorem 4.1. Let $k \in \mathbb{N}$ and $\operatorname{gcd}(q, p-1)=q$, then

$$
N\left(q^{k}, 1, p\right)=\frac{p^{q^{k}}-q p^{q^{k-1}}+q-1}{q^{k}(p-1)} .
$$

Proof. Let $n=q^{k}$ and let $r \in D_{n}$ with $m_{r}=1$. By [5, Theorem 3.5], we have

$$
N\left(q^{k}, 1, p\right)=\frac{1}{q^{k} \phi(1)} \sum_{\substack{r \in D_{n} \\ m_{r}=1}} \phi(r) .
$$

By Theorem 2.2 and properties of the Euler $\phi$ function, we get

$$
N\left(q^{k}, 1, p\right)=\frac{1}{q^{k}} \sum_{\substack{r \mid \frac{p^{n}-1}{p-1} \\ r \nmid p^{q^{k-1}}-1}} \phi(r)=\frac{1}{q^{k}}\left[\sum_{r \mid \frac{p^{n}-1}{p-1}} \phi(r)-\sum_{r \mid \operatorname{gcd}\left(\frac{p^{n}-1}{p-1}, p^{q^{k-1}}-1\right)} \phi(r)\right] .
$$

From Lemma 2.4 we know

$$
\operatorname{gcd}\left(\frac{p^{q^{k}}-1}{p-1}, p^{q^{k-1}}-1\right)=q \cdot \frac{p^{q^{k-1}}-1}{p-1},
$$

thus

$$
\begin{aligned}
N\left(q^{k}, 1, p\right) & =\frac{1}{q^{k}}\left[\frac{p^{q^{k}}-1}{p-1}-q \frac{p^{q^{k-1}}-1}{p-1}\right] \\
& =\frac{p^{q^{k}}-1-q\left(p^{q^{k-1}}-1\right)}{q^{k}(p-1)} \\
& =\frac{p^{q^{k}}-q p^{q^{k-1}}+q-1}{q^{k}(p-1)} .
\end{aligned}
$$

Theorem 4.2. Let $k \in \mathbb{N}, k \geq 2, \operatorname{gcd}(q, p-1)=q$, and a be a $q$-residue. Then

$$
N\left(q^{k}, 1, p\right)=N\left(q^{k}, a, p\right)
$$

Proof. Let $p-1=q^{j} s$, where $\operatorname{gcd}(s, q)=1$ and $j \in \mathbb{N}$. Since $p-1 \mid p^{q^{k-1}}-1$, this implies that $p^{q^{k-1}}$ is a multiple of $q^{j} s$. Furthermore, we can write $p^{q^{k-1}}-1=q^{i-1} s t$ where $\operatorname{gcd}(s, t)=1$, $\operatorname{gcd}(t, q)=1$, and $i-1>j$. By Corollary 2.4.1, the only prime divisor of $\operatorname{gcd}\left(\frac{p^{q^{k-1}}-1}{p-1}, p-1\right)$ is $q$, so $\operatorname{gcd}(s, t)=1$ and $i-1>j$. 
Now consider $p^{q^{k}}-1$. We have $p^{q^{k-1}}-1 \mid p^{q^{k}}-1$, hence we can write $p^{q^{k}}-1=q^{i}$ stu where $\operatorname{gcd}(u, q)=\operatorname{gcd}(s, t u)=1$. Note by Lemma 2.4, since $q^{i-1} \mid p^{q^{k-1}}-1$, we have $q^{i} \mid p^{q^{k}}-1$.

Let $n=q^{k}$ and $r \in D_{n}$ be a $q$-residue. Recall $m_{r} \mid p-1$, that is, $m_{r} \mid q^{j} s$. We also have $r=m_{r} d_{r}$ where $d_{r}=\operatorname{gcd}\left(r, \frac{p^{q^{k}}-1}{p-1}\right)=\operatorname{gcd}\left(r, q^{i-j} t u\right)$. By Theorem 3.1, $r$ being a $q$-residue implies $q^{j}$ does not divide $m_{r}$ (i.e., $m_{r}$ can have any power of $q$ except the maximum $q^{j}$ ).

First, let us evaluate $N\left(q^{k}, 1, p\right)$. If $m_{r}=1$, then $r \mid \frac{p^{q^{k}}-1}{p-1}$ by Lemma 2.1 and $r \nmid p^{q^{k-1}}-1$ because $r \in D_{n}$. In other words, $r \mid q^{i-j} t u$ and $r \nmid q^{i-1} s t$. We claim that there exists $u^{\prime} \neq 1$ such that $u^{\prime} \mid r$ and $u^{\prime} \mid u$. If not, then $\operatorname{gcd}(u, r)=1$ implies $r \mid q^{i-j} s t$. But then $r \mid q^{i-1} s t$, which is a contradiction. Thus, $r=q^{\ell} t^{\prime} u^{\prime}$ for some $\ell \in\{0, \ldots, i-j\}, t^{\prime}\left|t, u^{\prime}\right| u, u^{\prime} \neq 1$. Now we have

$$
\begin{aligned}
N\left(q^{k}, 1, p\right) & =\frac{1}{q^{k} \phi(1)} \sum_{\substack{r \in D_{n} \\
m_{r}=1}} \phi(r) \\
& =\frac{1}{q^{k}} \sum_{\substack{\ell \in\{0, \ldots, i-j\} \\
t^{\prime}\left|t, u^{\prime}\right| u, u^{\prime} \neq 1}} \phi\left(q^{\ell}\right) \phi\left(t^{\prime}\right) \phi\left(u^{\prime}\right) \\
& =\frac{q^{i-j} t(u-1)}{q^{k}} \\
& =\frac{t(u-1)}{q^{k-i+j}} .
\end{aligned}
$$

Now suppose $m_{r} \neq 1$, say $m_{r}=q^{b} s^{\prime}$ for some $b \in\{0, \ldots, j-1\}$ and $s^{\prime} \mid s$. Note that $b \leq j-1$ implies $q^{j} \nmid m_{r}$ and so $q^{i} \nmid r$. We claim that there exists $u^{\prime} \mid u, u^{\prime} \neq 1$, such that $u^{\prime} \mid r$. If not, $\operatorname{gcd}(u, r)=1$ and $r \mid p^{q^{k}}-1$ implies $r \mid q^{i} s t$. But $q^{i} \nmid r$, so $r \mid q^{i-1} s t$, contradicting $r \in D_{n}$. Thus, $r=q^{\ell} s^{\prime} t^{\prime} u^{\prime}$ for some $\ell \in\{0, \ldots, i-1\}, s^{\prime}\left|s, t^{\prime}\right| t, u^{\prime} \mid u, u^{\prime} \neq 1$. There are two cases to consider: $m_{r}=s^{\prime}$ and $m_{r}=q^{b} s^{\prime}$ for some $b \in\{0, \ldots, j-1\}$.

Case 1: $\left(m_{r}=s^{\prime}\right)$ In this case $\ell \in\{0, \ldots, i-j\}$. It follows that

$$
\begin{aligned}
N\left(q^{k}, a, p\right) & =\frac{1}{q^{k} \phi\left(s^{\prime}\right)} \sum_{\substack{r \in D_{n} \\
m_{r}=s^{\prime}}} \phi(r) \\
& =\frac{1}{q^{k} \phi\left(s^{\prime}\right)} \sum_{\substack{\ell \in\{0, \ldots, i-j\} \\
t^{\prime}\left|t, u^{\prime}\right| u, u^{\prime} \neq 1}} \phi\left(q^{\ell}\right) \phi\left(s^{\prime}\right) \phi\left(t^{\prime}\right) \phi\left(u^{\prime}\right) \\
& =\frac{q^{i-j} t(u-1)}{q^{k}} \\
& =\frac{t(u-1)}{q^{k-i+j}} \\
& =N\left(q^{k}, 1, p\right) .
\end{aligned}
$$

Case 2: $\left(m_{r}=q^{b} s^{\prime}\right)$ We claim $\ell=i-j+b$ for some $b \in\{1, \ldots, j-1\}$. If $\ell \leq i-j$, then $d_{r}=\operatorname{gcd}\left(r, \frac{p^{q^{k}}-1}{p-1}\right)=\operatorname{gcd}\left(q^{\ell} s^{\prime} t^{\prime} u^{\prime}, q^{i-j} t u\right)=q^{\ell} t^{\prime} u^{\prime}$ implies $b=0$, a contradiction. Hence, $\ell>i-j$ and we can write $\ell=i-j+b$ for some $b \in\{1, \ldots, j-1\}$. It follows that 


$$
\begin{aligned}
N\left(q^{k}, a, p\right) & =\frac{1}{q^{k} \phi\left(q^{b} s^{\prime}\right)} \sum_{\substack{r \in D_{n} \\
m_{r}=q^{b} s^{\prime}}} \phi(r) \\
& =\frac{1}{q^{k} \phi\left(q^{b} s^{\prime}\right)} \sum_{\substack{t^{\prime}\left|t, u^{\prime}\right| u, u^{\prime} \neq 1\\
}} \phi\left(q^{i-j+b}\right) \phi\left(s^{\prime}\right) \phi\left(t^{\prime}\right) \phi\left(u^{\prime}\right) \\
& =\frac{1}{q^{k} \phi\left(s^{\prime}\right)\left(q^{b}-q^{b-1}\right)} \sum_{t^{\prime}\left|t, u^{\prime}\right| u, u^{\prime} \neq 1}\left(q^{i-j+b}-q^{i-j+b-1}\right) \phi\left(s^{\prime}\right) \phi\left(t^{\prime}\right) \phi\left(u^{\prime}\right) \\
& =\frac{\left(q^{i-j+b}-q^{i-j+b-1}\right) t(u-1)}{q^{k}\left(q^{b}-q^{b-1}\right)} \\
& =\frac{t(u-1)}{q^{k-i+j}} \\
& =N\left(q^{k}, 1, p\right) .
\end{aligned}
$$

It is worthwhile to note that Theorem 2.5, Theorem 4.1, and Theorem 4.2 each produce a formula for $N\left(q^{k}, a, p\right)$ that depends only on whether or not $a$ is a $q$-residue. In particular, $N\left(q^{k}, a, p\right)$ takes only one or two distinct values for a given $q^{k}$ and $p$. The following relationship is particularly interesting:

Corollary 4.2.1. Let $\operatorname{gcd}(q, p-1)=q$ and $k \in \mathbb{N}$. If a is a non q-residue and $b$ a $q$-residue in $\mathbb{Z}_{p}^{*}$, then

$$
N\left(q^{k}, a, p\right)-N\left(q^{k}, b, p\right)=N\left(q^{k-1}, a, p\right) .
$$

While this corollary shows that the difference between $N\left(q^{k}, a, p\right)$ and $N\left(q^{k}, b, p\right)$ increases as $k$ increases, we will show that the ratio $\frac{N\left(q^{k}, a, p\right)}{N\left(q^{k}, b, p\right)}$ approaches one. If $\operatorname{gcd}(p-1, q)=1$, then by Theorem 2.5 the constant terms of all monic irreducible polynomials are uniformly distributed. Thus, the ratio $\frac{N\left(q^{k}, a, p\right)}{N\left(q^{k}, b, p\right)}$ equals one for any $a, b \in \mathbb{Z}_{p}^{*}$.

Notice that by Theorem 3.2 the number of irreducible monic polynomials with constant term $a$, where $a$ is not a $q$-residue and $\operatorname{gcd}(p-1, q)=q$, is given by

$$
\frac{p^{q^{k}}-1}{q^{k}(p-1)}
$$

and when $b$ is a $q$-residue, the number is

$$
\frac{p^{q^{k}}-q p^{q^{k}-1}+q-1}{q^{k}(p-1)} .
$$

Hence the ratio

$$
\frac{N\left(q^{k}, a, p\right)}{N\left(q^{k}, b, p\right)}=\frac{p^{q^{k}}-1}{q^{k}(p-1)} \cdot \frac{q^{k}(p-1)}{p^{q^{k}}-q p^{q^{k}-1}+q-1}
$$

approaches one as $k$ approaches infinity.

This shows us that the proportions of constant terms of monic irreducible polynomials are asymptotically equal, as their limits show a uniform distribution among the constant terms. 


\section{References}

[1] Krizek, M., Luca, F., \& Somer, L. (2001). 17 Lectures on Fermat Numbers, CMS Books in Mathematics, Springer-Verlag, New York.

[2] Lidl, R., \& Niederreiter, H. (1994). Introduction to Finite Fields and Their Application, Revised edition, Cambridge University Press, Cambridge.

[3] Niven, I., Zuckerman, H., \& Montgomery, H. (1991). An Introduction to the Theory of Numbers, 5th edition, Wiley and Sons, Inc., New York.

[4] Rubinstein, M., \& Sarnak, P. (1994). Chebyshev's Bias, Experimental Mathematics, 3, 173-197.

[5] Yucas, J. L. (2006). Irreducible polynomials over finite fields with prescribed trace/prescribed constant term, Finite Fields and Their Appl, 12, 211-221. 